\title{
Open Vehicle Sketch Pad Aircraft Modeling Strategies
}

\author{
Andrew S. Hahn ${ }^{1}$ \\ NASA Langley Research Center, Hampton, VA, 23681
}

\begin{abstract}
Geometric modeling of aircraft during the Conceptual design phase is very different from that needed for the Preliminary or Detailed design phases. The Conceptual design phase is characterized by the rapid, multi-disciplinary analysis of many design variables by a small engineering team. The designer must walk a line between fidelity and productivity, picking tools and methods with the appropriate balance of characteristics to achieve the goals of the study, while staying within the available resources. Identifying geometric details that are important, and those that are not, is critical to making modeling and methodology choices. This is true for both the low-order analysis methods traditionally used in Conceptual design as well as the highest-order analyses available. This paper will highlight some of Conceptual design's characteristics that drive the designer's choices as well as modeling examples for several aircraft configurations using the open source version of the Vehicle Sketch Pad (Open VSP) aircraft Conceptual design geometry modeler.
\end{abstract}

\section{Nomenclature}

$\begin{array}{ll}3 D & =\text { three dimensional } \\ C A D & =\text { computer aided design } \\ C F D & =\text { computational fluid dynamics } \\ D O E & =\text { design of experiments } \\ d p i & =\text { dots per inch } \\ G A & =\text { General Aviation } \\ G U I & =\text { graphical user interface } \\ I G E S \quad= & \text { Initial Graphics Exchange Specification } \\ \text { OML } & =\text { outer mold line } \\ \text { Open } V S P & =\text { open source version of the Vehicle Sketch Pad aircraft Conceptual design geometry modeler } \\ \text { PAV } & =\text { personal air vehicle } \\ \text { ROM } & =\text { reduced order model } \\ \text { STL } & =\text { stereolithography file format }\end{array}$

\section{Introduction}

$\mathrm{I}$ deally, we wouldn't need to bother at all with the Conceptual or Preliminary design phases. In an unlimited world every possible combination of configuration and technology could be designed in detail, built, tested, and compared. Unfortunately, we live in a world of resource limits far below those of our imaginations. These real world limitations have forced us to find other, less expensive means for the design of aircraft. The basic phases of the design process are Conceptual, Preliminary, and Detailed. It is a matter of judgment as to where one ends and the next begins, but they roughly correspond to the functions of design space exploration, risk reduction, and production.

Since these phases serve different functions, they have different characteristics that profoundly shape their processes and methods. The Conceptual design phase is characterized by the rapid, multi-disciplinary analysis of many design variables by a small engineering team. These characteristics put a premium on speed, flexibility, and productivity. Once resource limitations are accepted, what is lost by necessity is fidelity; however, there are details that have a very small influence on metrics, there are details that don't discriminate between choices, there are details that require a great deal of time and effort to engineer only to have the result equal a customary penalty, and there are details that have a large influence on metrics. The careful selection of which details to neglect and which to keep, enables exploring a design space with dramatically reduced degrees of freedom while maintaining a relatively

\footnotetext{
${ }^{1}$ Aerospace Engineer, Aeronautics Systems Analysis Branch, Mail Stop 442, AIAA Member.
} 
low loss in fidelity. More importantly, there may be no loss in the ability to discriminate between design choices, and therefore, the ability to rank them. This means that the best design choices may be correctly made despite having lesser fidelity, or somewhat greater uncertainty. As the design is refined in the Preliminary design phase, the risk associated with uncertainty is reduced, but the choices deemed to be the best in the Conceptual design phase will ideally remain so.

Over the years, a multi-order, multi-discipline design process has evolved to allow broad, affordable exploration of the design space, enabling the analysis of unconventional configurations and advanced technology suites to be considered during the Conceptual design phase. The starting point for the process, Open Vehicle Sketch Pad (Open VSP), is a parametrically driven, open source geometry software package that is intended to make modeling details relevant to aircraft design easy to implement, while sacrificing some of the flexibility that a computer aided design (CAD) package can provide. ${ }^{1}$ This tradeoff between productivity and fidelity is most appropriate at the Conceptual design phase, but may also hold to a lesser degree into the Preliminary design phase. The parameterization in Open VSP maintains certain design intents that make analysis using high-order methods, such as computational fluid dynamics (CFD), productive despite large changes in the geometry. This paper will present best practices for aircraft geometry modeling that strike an appropriate balance between productivity and fidelity, along with tips on how to avoid common modeling pitfalls.

\section{Aircraft Modeling Using Open Vehicle Sketch Pad}

The statistician, George E. P. Box, is credited with saying, "All models are wrong but some are useful."2 It is this simple observation that gives comfort to the aircraft designer in the Conceptual design phase when faced with the daunting task of designing the many aircraft required in the course of a design study.

Clearly, designing just one aircraft to the level of detail and fidelity required for production is far beyond the resources available. Designing the hundreds of aircraft embodying the multi-disciplinary variations of interest required for a study only compounds the problem. Given the practical limitations on the available effort, Conceptual design has relied on reduced order models (ROMs), primarily through regressions of data that describe real aircraft. These regressions have yielded so called "handbook" methods and parameterizations that are familiar to the aerospace engineer. These methods are very useful in that the reduction in the degrees of freedom, or order, make defining a new aircraft, or modeling an existing aircraft, relatively easy. The amount of data required by the analysis is manageable, even with a simple text-based input. Individual function evaluations are fast, allowing many designs to be created and compared. The integration of these tools with an optimizer, enables a more efficient search through the design space. Since these methods are based on real aircraft, they embody a multitude of real world constraints and inefficiencies that may not be directly modeled by the parameterization. These methods do not yield what a particular design's characteristics are, as much as indicate what the design's characteristics should customarily be. Reliance on a database of real aircraft leveraged the many thousands of man-hours required to fully design the aircraft that made up the database.

This approach works well when investigating conventional configurations and sensitivities to modest improvements in technologies. This same approach may quickly lose validity as the designer strays from the aircraft types that make up the database. It has long been a desire of conceptual aircraft designers to augment the methods available with analyses that are sensitive to more degrees of freedom, or are higher-order. These analyses should allow evaluations of unconventional configurations and greater changes in individual technologies than is possible with the traditional handbook methods. Unfortunately, higher-order analyses require larger amounts of very specific information than the low-order analyses do, and this information is difficult for the designer to supply manually.

Open VSP was created to manage this information and to bridge the gap between the "handbook" methods and higher-order analyses. In order to do this, Open VSP adds some new degrees of freedom, or design variables, to the traditional geometry descriptors as well as a graphical user interface (GUI) for visualization. Open VSP also treats its internal representation of the geometry as the source of customized representations, or meta-geometries, suitable for supported analyses. It is this ability to translate the internal geometry representation into a meta-geometry that an individual analysis understands that allows the designer to choose a tool that best suits the problem at hand. Whereas all models are wrong, some are useful and the designer has the privilege of choosing the tradeoff between fidelity and productivity, as well as the responsibility of understanding the pros and cons of that choice. It is then up to the designer to model the aircraft in sufficient detail to feed the chosen analysis. 


\section{A. Creating The Open VSP Geometry}

There are two basic kinds of models created in Open VSP. The first is the "clean sheet" design in which the parameters are all chosen by the designer using Open VSP. In this case, there is no other geometry and so this model is considered definitive. In one sense, this is the easiest model to build at the start since each parameter may be chosen without regard to some standard of comparison. The only "unknowns" are the values of parameters that the designer has not explicitly chosen; however, whereas the designer has complete control, he also has complete responsibility. In the end, the performance of this design will depend on the amount of analysis that is put into it. One example of this kind of model is the two seat personal air vehicle (PAV), of which a rapid prototyped wind tunnel model is shown in Fig. 1.

The second basic kind of model is the "match" design. This kind of model is created for either analysis validation or calibration. In this case, there is some other standard of comparison, be it a real

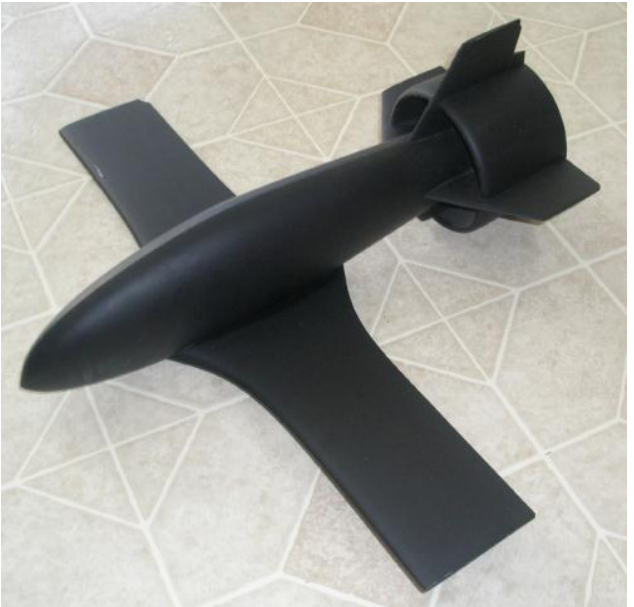

Figure 1. Two seat personal air vehicle wind tunnel model. aircraft or a geometry from a different modeler such as CAD. It takes significantly more effort to produce a model that is as good of a representation as possible. Usually, the only geometric information available is limited tabular data and a three-view drawing. There are different ways of building this kind of model, but the preferred way is to gather the most accurate information and then expend some effort to derive the parameters that Open VSP needs to create the model. Frequently, the data are self inconsistent and it requires expert judgment to come up with the best set.

When creating the "match" design, often the available tabular data is far from adequate, making the use of a three-view drawing very helpful. It is unusual for well dimensioned drawings to be publicly available though, making measuring the available drawing necessary. If the drawing is printed, it should be scanned at a high resolution, such as 600 dots per inch (dpi). If the drawing is a graphic file, then it must be used at its native resolution. In either case, import the graphic into a drawing program to perform dimensioning. The first thing to check is that the graphic is of the same dimensionality in both the vertical and horizontal directions. It is surprising how often the graphic is distorted. If the graphic is distorted, simply scale one of the directions independent of the other until they are consistent. This operation benefits greatly from having at least one dimension on the graphic in each direction. Whereas it is possible to import the graphic directly into the background of Open VSP and adjust parameters to match "by eye," the preferred approach benefits from the ability to zoom into the graphic to more precisely measure the parameters of interest. The only task where using the background capability of Open VSP is best is at the end, when adjusting tangent strengths on the fuselage component to match curvature. This is because there is no good way to predetermine these values from the graphic. One thing to be aware of is that the wing area generally listed is a reference area and may not be the same as what is measured from the drawing. This usually requires that the total wing area in Open VSP be different in order to best match the drawing. Open VSP is aware of this and allows the designer to specify a reference area that is different, but since the reference area is somewhat arbitrary, it is not automatically updated if the wing design is changed. Creating a dimensioned drawing and consistent tabular data usually requires about four hours to complete. Unfortunately, the information provided is generally not adequate for high-order analysis as crucial parameters, such as airfoil coordinates and wing twist distributions, are usually missing. This resulting model is a good basis for low-order analysis, and with some additional design work, may be a good basis for highorder analysis.

It is becoming more common to have an outer mold line (OML) provided in a non-parameterized form such as an airfoil stack, or an Initial Graphics Exchange Specification (IGES) file, or a stereolithography (STL) file. All

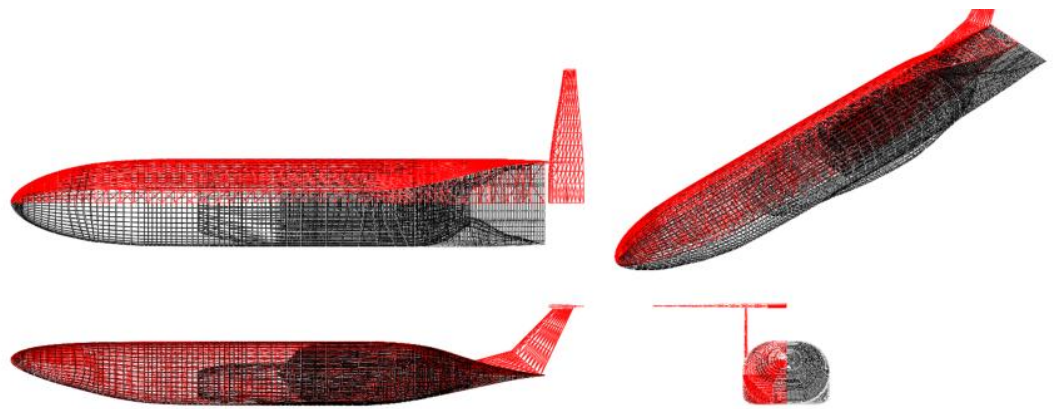

Figure 2. STL half model (red) used as a template for a parameterized fuselage component (black). 
of these files may be imported into a drawing program and be dimensioned as the three view drawing was, but the STL file may also be imported directly into Open VSP and used as a three dimensional (3D) template. These kinds of files do contain all of the information necessary for high-order analysis, but will require some additional effort to extract it. Currently, parameterization is a completely manual process that yields good fidelity, but there is a plan to add an assisted fitting capability to Open VSP for the STL component. It is too difficult to make parameterization of a component automatic in general, but it would still be very useful for Open VSP to find the optimal settings for parameters specified by the designer to minimize the error between the original, non-parameterized, and the manually parameterized components. Of course, the initial quality of the manually defined component and the ability of the parameters to fit the original surface will have a strong influence on the final result. Since the original, non-parameterized surface usually comes from a high-order analysis, it is possible to run the new parameterized component through the same analysis to quantify the resulting modeling error. Figure 2 shows an example of using an STL half model as a template for the manually parameterized fuselage, which is made up from several components.

\section{B. Component Selection}

Open VSP currently provides 12 component types for the designer to choose from. Whereas each component has its strengths and weaknesses, the four that may be used almost exclusively are the BLANK, MS WING, FUSE2, and PROP components.

The BLANK component at first seems to be too simple to be of use. There is no surface or shape associated with it. The main use of the BLANK is to serve as the parent to a multi-component subsystem that the designer wishes to translate, rotate, show, or hide as a group. Essentially, the BLANK serves as a coordinate system local to its children.

The MS WING, or multi-section wing, component is used to model an aircraft's wing-like parts. This component is obviously intended for modeling wings, but it also may be the most appropriate choice for tails, pylons, struts, antennae, etc. Complex planforms are modeled by adding trapezoidal sections as needed, the key concept being "as needed." There is a natural tendency of many designers to strive for the highest fidelity possible, producing components with many slightly varying sections. This is understandable, but it may not be the best choice in the Conceptual design phase. As the number of sections increases, the number of design variables multiplies, making an optimization or design of experiments (DOE) less tractable. The designer should also bear in mind the characteristics of the analysis that is being used. Often, low and medium-order analyses are insensitive to fine details, rendering subtleties an additional burden for no additional benefit. Bearing this in mind, it is good practice to have a simple trapezoidal reference wing for the low-order analysis and a separate multi-section wing with a minimal number of sections for the medium and high-order analyses.

The FUSE2, or second generation fuselage, component is used to model an aircraft's bodies. This component is obviously intended for modeling the fuselage, but it is surprisingly versatile. FUSE2 can be used for almost any part that is not wing-like. It has been used for pods, fairings, cockpit canopies, landing gear struts and tires, piston engines, drive shafts, flow-through turbofan nacelles, electronics boxes, cargo containers, seats, and even a human manikin. FUSE2 was created because the original FUSE component was simply incapable of producing many common fuselage shapes in the longitudinal direction.

In order to provide the necessary degree of control, the idea of Bézier curves to specify the longitudinal shape between user-defined, or hard, cross sections was borrowed from the cross section definition. Since Bézier curves are not a standard aircraft engineering parameterization, this is an area that seems to cause the most confusion. Bézier curves are specified by points that the curve must go through, and at each of those points, the curve shape is controlled by a tangent angle and tangent strength vectors on either side. Figure 3 shows a generic Bézier curve where the squares are the curve points and the tangent lines represent the strength vectors. The real power of this parameterization is that the curves between cross sections are guaranteed to be smooth and continuous to second order with a maximum of one inflection. These are characteristics that will tend to produce good aerodynamic shapes.

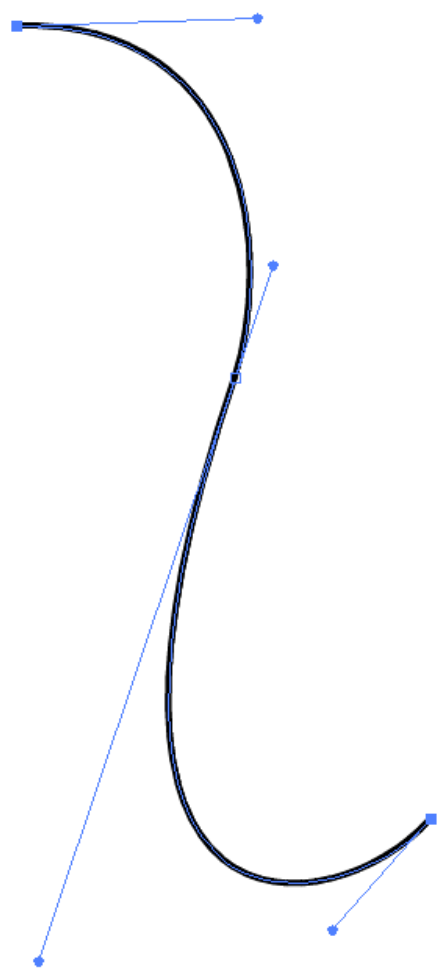

Figure 3. Bézier curve example. 
This is also an example of how Open VSP's parameterization was chosen to preserve design intent; in this case, fuselages should generally be aerodynamic.

As with the wing, less is more. There is a natural tendency of designers to try to control the shape of the fuselage through a large number of hard cross sections. Not only does this defeat the benefits of parameterization by increasing the number of design variables, but it is very likely to generate surface undulations, or higher order ringing, that we want to avoid.

The last of the most-used components is not really a separate component at all. The PROP, or propeller component, is obviously intended for modeling propellers, but it is also used to model fans, compressors, and turbines as well. The PROP component looks to the user like it is a separate component, but in actual fact, it is simply a multi-section wing with a different user interface. Instead of the designer performing the tedious task of creating a blade using the MS WING component, making copies, translating, and rotating them to produce the propeller, Open VSP accepts input that makes sense for this application and is easy to supply by the designer, and then performs all of the necessary operations behind the scenes. This component is just one example of how the parameterization promotes ease of use.

\section{General Modeling Strategies}

This section suggests general strategies to help designers produce geometry models with the best balance between productivity, fidelity, and the preservation of design intent, as well as how to avoid the most common problems through best modeling practices.

\section{Less Is More}

In general, the designer should strive to use the minimum number of parameters to specify the geometry of interest. If a component appears to be overly complex, it probably is so. For example, a typical fuselage may be represented by as few as four hard cross sections. Figure 4 shows a fuselage modeled with FUSE2. The upper

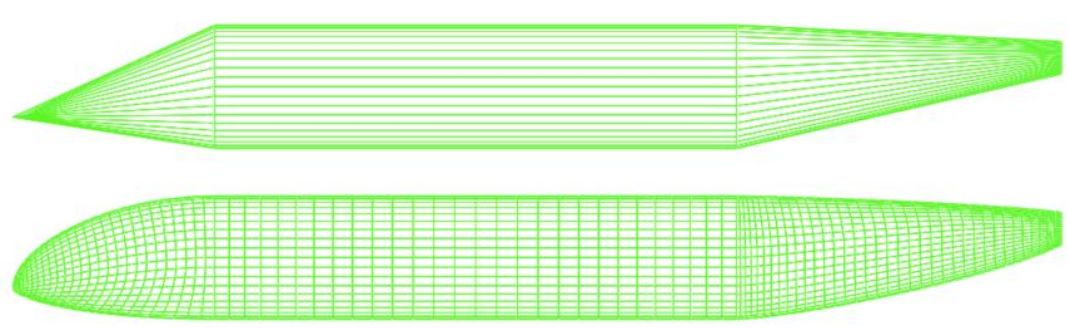

Figure 4. Fuselage model illustrating hard and soft cross sections. model is the same as the lower, except that it has no interpolated, or soft, cross sections. There are only four hard cross sections. The first cross section is simply a point, the two intermediates are ellipses, and the last is an ellipse with a width of zero. When compared with the lower fuselage, it can be seen that all of the curvature in the longitudinal direction is due to the Bézier curves determining the shapes of the soft cross sections. The natural tendency of designers new to Open VSP is to specify hard cross sections in the curved regions, particularly if they have cross section stacks available to them. Unfortunately, each cross section requires its corresponding tangent angle and strengths set to values consistent with the original, presumably smooth surface. The odds of this actually occurring are quite low and it would be much better to model the stack with no interpolated cross sections, make a copy, delete the cross sections in the curved parts, and then adjust the upper, lower, and side tangents to best pass along the edges of the original stack. Another option for setting the tangent values is to adjust them with a picture or drawing of the aircraft being modeled in the background, as previously noted.

\section{Remove Details That Either Have Minimal Impact Or Do Not Discriminate Between Designs}

There are many details that are very important in the late Preliminary and Detailed design phases that are not worth modeling explicitly in the Conceptual design phase. For example, analyzing the drag increment due to the various sources of excrescence in the Conceptual design phase would be extremely difficult, relatively inaccurate,

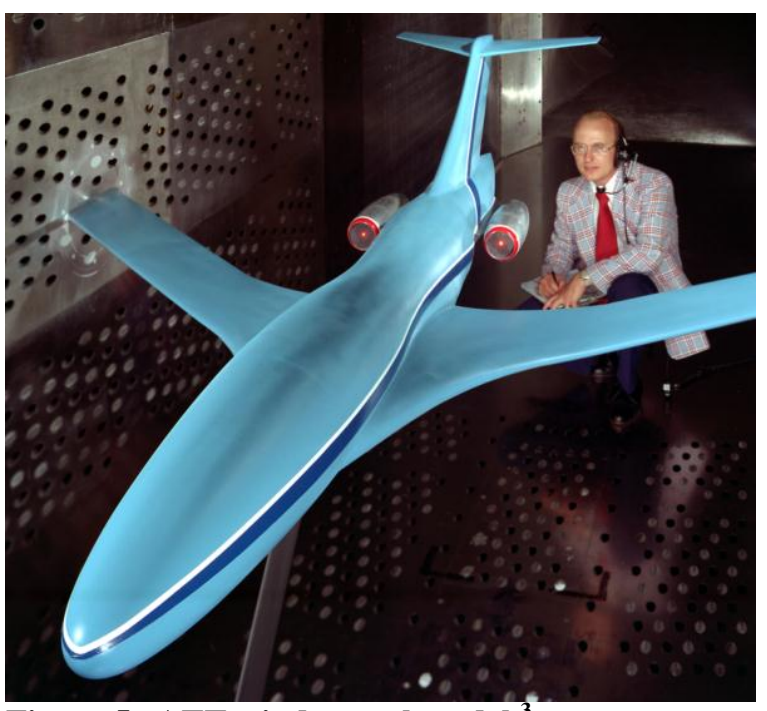

Figure 5. ATT wind tunnel model. 
and not significantly different from one design to the next. Standard practice is to assess a constant, customary drag increment that is added to the analysis results.

Similarly, modeling a cockpit on an airliner is usually not worth the effort in the Conceptual design phase. Unless there is some reason to believe that this particular instance of the cockpit integration would have significant impact and discriminate between alternatives in the study, the drag increment is best handled as the excrescence drag is. The natural tendency of designers, especially when using highorder analysis such as CFD, is to build in as much geometric fidelity as possible. However, unless they also design the cockpit to at least the level done in the Preliminary design phase, then this "fidelity" is illusory. No one would argue that wind tunnel tests are low fidelity, and yet it is common practice to test airliner models without cockpits for the reasons stated earlier (Fig. 5-7).

Another common issue is whether to model a "double bubble" cross section explicitly as two separate circular fuselages or to simply model with an ellipse on a single fuselage. This is a matter of judgment, but the designer should weigh the increased difficulty of modeling and manipulation against the loss in fidelity. If the diameter differences are slight, as they are on a McDonnell-Douglas MD-80 or Boeing 737, then the loss in fidelity is slight with the ellipse. However, if the differences are great, as in the upper deck of the Boeing 747 or the Boeing Stratocruiser, then the extra effort may well be worth it.

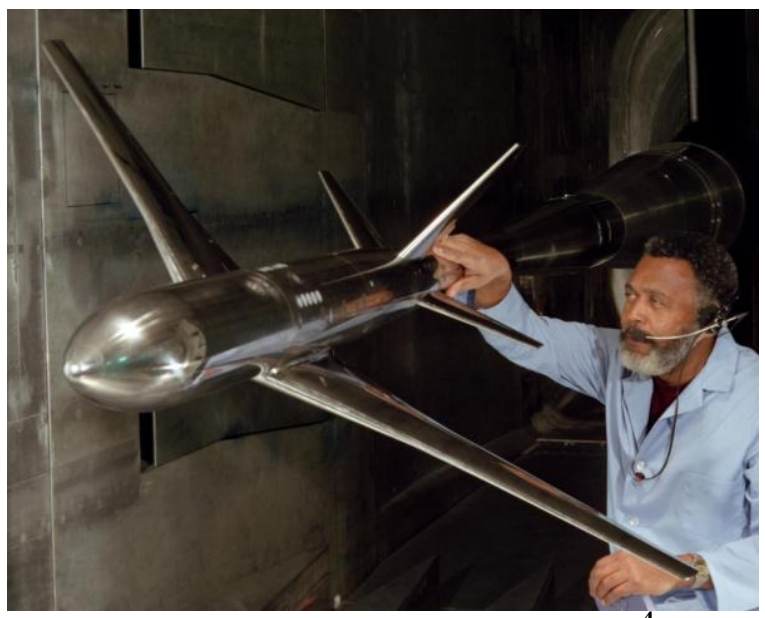

Figure 6. Pathfinder I wind tunnel model.

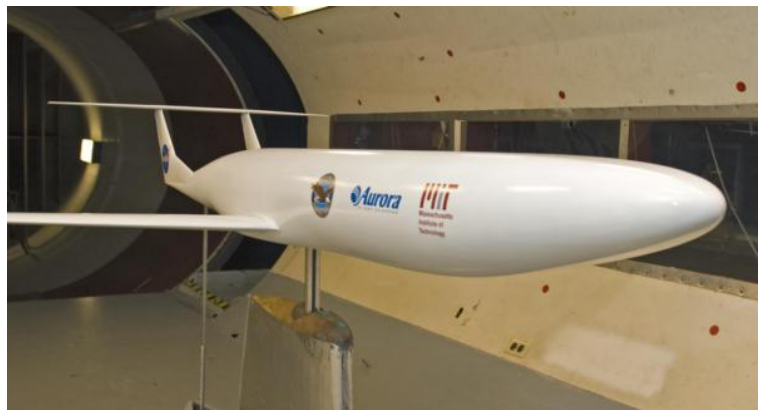

Figure 7. MIT D8 wind tunnel model.

\section{Remove Details That Require A Large Design Effort To Achieve A Customary Penalty}

There are some details that have significant impact and so can not be ignored in the Conceptual design phase, but that are also very difficult to design. Often a great deal of design and analysis effort is expended with the final result being that the penalty associated with the detail is minimized to some customary value. For example, most airliners have engine pods hanging from the wing on pylons. A poor pylon design can not be tolerated, and it takes substantial effort to design a pylon for a given aircraft to minimize the penalty. Whereas there may be significant difficulties from one particular installation to the next that may, or may not, be resolved, it is impractical to expend the level of design effort required for each of the hundreds of aircraft needed in a design study. This is particularly evident when one realizes that the vast majority of the aircraft designed for the study will not be in the optimal neighborhood. Simply

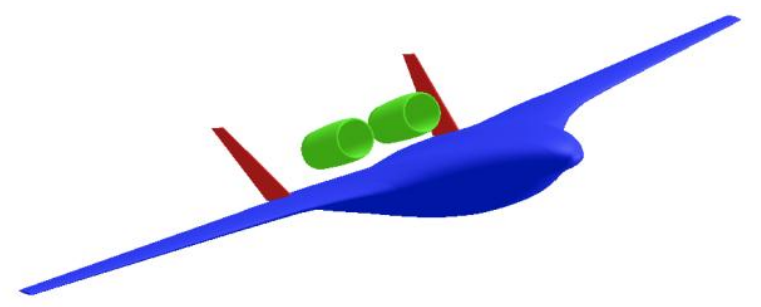

Figure 8. Blended Wing Body w/o pylons. put, the vast majority of the effort expended in pylon design in a study is wasted because most of the aircraft designed are losers. Low-order analysis already deals with this problem by having customary penalties applied during the drag build-up, but what can be done in a high-order analysis where the design provided is analyzed for how it performs, not how it should be capable of performing? Our approach for pylons is simply to leave them off during the high-order analysis and apply the same customary penalty that the low-order analysis would. This is one non-obvious advantage that CFD has over wind tunnels. CFD is perfectly happy to have the engine pod fly in formation with the wing with no physical means of support (Fig. 8). The study will narrow the myriad of possible designs to a small neighborhood of competitive designs. Then, the necessary design effort may be expended once to verify that the customary penalty is indeed possible to attain. 


\section{Build Complex Shapes From Multiple Simple Shapes}

The components provided in Open VSP are surprisingly flexible, despite being parameterized. The FUSE2 component is especially flexible in that more degrees of freedom are available with every additional hard cross section. It is possible to build up complex fuselage shapes through specifying any number of hard cross sections as desired. Some models, such as many light General Aviation (GA) aircraft, are so irregular that they may require this approach.

Figure 9 shows a typical GA Aircraft, the Cessna 182, with a fuselage modeled solely by hard cross sections. Whereas it is important to be able to model this fuselage at all, the number of cross sections needed makes it tedious to implement even the smallest of design changes. For example, widening the cockpit area would require modifying no less than 34 values. Also, matching the tangent angles and strengths to a smooth OML becomes nearly impossible, as was mentioned earlier. This approach should be avoided in general as it basically negates most of the benefits of parameterization.

Other models, such as most airliners and business jets, are

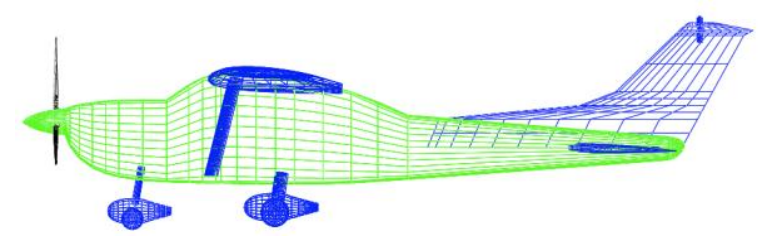

Figure 9. Cessna 182 model.

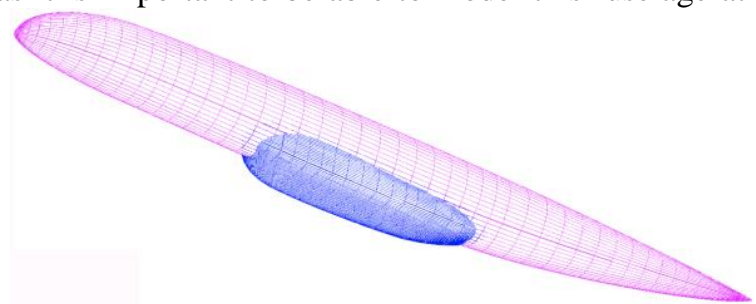

Figure 10. Airliner fuselage and fairing. better modeled through multiple simple components. Figure 10 shows an airliner fuselage with a wing fairing. In this case both the fuselage and fairing are modeled using only four hard cross sections. Modifications are much less tedious and may be made independently. For example, the cabin width could be changed by modifying only two values, or the position of the fairing could be changed with only one value. Open VSP will then find the intersection automatically, correctly accounting for surface areas and volumes.

\section{Avoid Open Components}

All of the default components in Open VSP are individually closed, or watertight. All unions of these components are also watertight. This is important as all of the meta-geometries generated for analysis must also be watertight. The only components that the designer can modify so that they are no longer watertight are FUSE and FUSE2. The easiest way to ensure that these components remain watertight is to not modify the first or last cross sections, which are points by default.

There are times; however, when modifying these cross sections is very useful. One example is of the last cross section of the fuselage in Figure 4. In this case, the last cross section was an ellipse of $3 \mathrm{ft}$. height and $0 \mathrm{ft}$. width, modeling a wedge-like closure. Since the width was zero, the fuselage remained watertight.

Another time when modifying the first and last cross sections is useful is when modeling flow-through turbofan nacelles. Whereas the DUCT component does an excellent job of modeling nacelles that are axisymmetric, many nacelles are far from axisymmetric. Modeling these nacelles requires the flexibility of FUSE2, but there is a problem. FUSE2 has only one surface that can be considered wetted. If the first and last cross sections are opened to model the inlet and nozzle, then the component is no longer watertight and

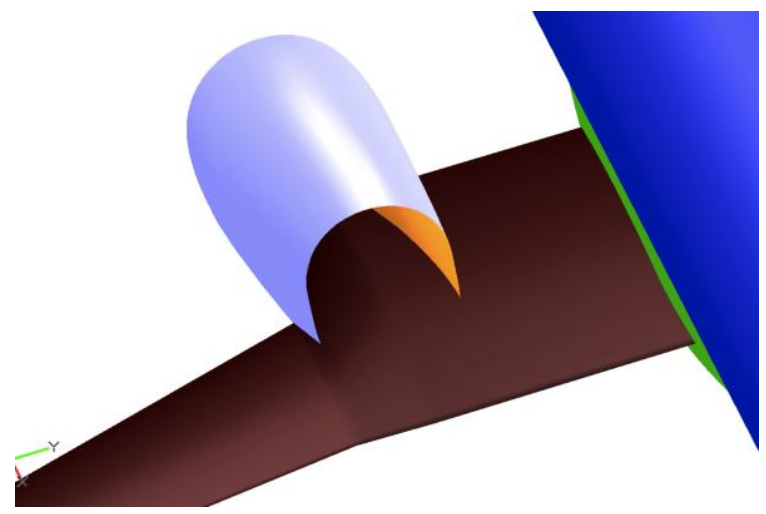
Figure 11. Non-axisymmetric flow-through engine nacelle. all analyses will fail. In order to enable this very important modeling capability, Open VSP has one very specific exception. If the outer nacelle is modeled with FUSE2, then the designer may make a copy and change any parameters to model the inner wall of the nacelle. As long as the first and last cross sections are exactly the same, then Open VSP will recognize these two components as if they were one watertight component. This scheme is also very useful because when triangulating the surface for high-order analyses, Open VSP will tag every triangle with the appropriate component number, allowing flow solvers to apply different bookkeeping or boundary conditions to the inside or outside. Figure 11 shows an example of a very non-axisymmetric nacelle modeled this way, applied to an Over-Wing Nacelle concept. 


\section{Avoid Coincident Components}

Perhaps the most common, and most easily avoided problem when modeling in Open VSP is when two components' surfaces share the exact same space. In these cases, the intersection routine has difficulty distinguishing the division between the surfaces, causing the intersection to fail.

The most common reason that this occurs is that more than one component will share the same starting or ending point. This can occur when the designer intends for a configuration, such as a cruciform tail, to be perfectly aligned. It is good practice to get in the habit of giving any components that may fall into this category a little nudge, appropriate to the scale of the model, such that any analysis is not significantly impacted, but so that the coincidence is prevented. This preventative practice is time saving when the difficulty of determining which components are causing the failure is factored in.

Another related problem that frequently comes up is when the designer intends to butt two components up against each other or have two components blend together tangentially. Once again, surface points become infinitesimally close to each other, making it hard for the intersection routine to find a smooth intersection path between them. The key practices to avoid these problems are to ensure that any components that are to be intersected clearly share a significant amount of volume and that the surfaces have some finite angle with respect to each other.

Figure 12 illustrates multiple instances of bad practice. In this model, there is an attempt to butt the wings and tail up against the fuselage and fairing. There is no reason to do this, as Open VSP doesn't care how much of a component is buried or even if multiple components share the same volume. Open VSP will eliminate any surface or volume that is not wetted, so there is no chance that they will be multiply bookkept. Attempting this creates problems, as intersections may become ambiguous and gaps may open up creating very narrow crevices. These peculiarly shaped crevices don't really exist and may be difficult to fill with a volume grid in a CFD analysis. Also,

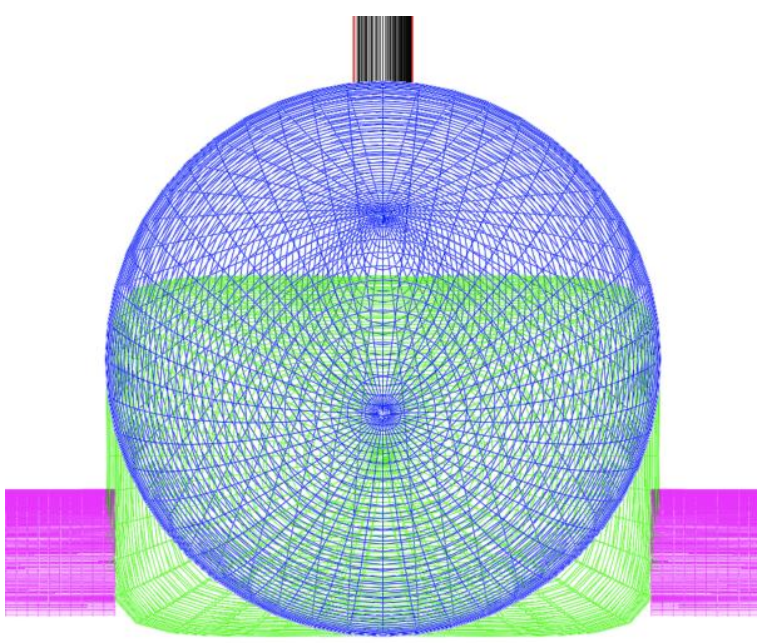

Figure 12. Bad practice.

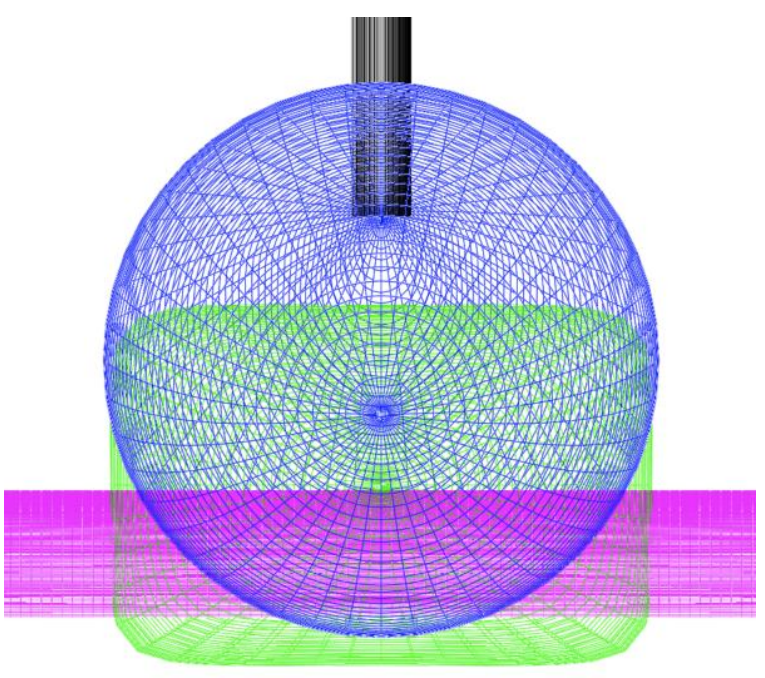

Figure 13. Good practice.

the fairing approaches the fuselage tangentially both on the bottom and on the sides creating further intersection ambiguity.

Figure 13 suggests how the model should be constructed. The wing goes all of the way to the centerline, the tail is substantially buried in the fuselage, and the fairing does not approach the fuselage tangentially. Not only was Open VSP able to determine the wetted surface areas and volumes, but having margin allowed minor changes to be made to the fuselage without creating any new degeneracies.

\section{Conclusion}

Conceptual design is the earliest of the design phases, with the purpose of design space exploration. It is characterized by rapid, multi-disciplinary analysis of many design variables by a small engineering team. This puts a premium on productivity, and necessarily reduces the appropriate fidelity to make a given study tractable. Open Vehicle Sketch Pad (Open VSP) was developed to help the conceptual designer bridge the gap between traditional low-order analysis and high-order analysis with the goal of expanding Conceptual design beyond historical databases. Introducing high-order analysis brings with it new issues that need to be addressed; however, there are 
several basic strategies that may be employed to allow effective use of these analyses. Several of these strategies, specific to Open VSP geometry modeling have been presented, along with corresponding example cases.

\section{Acknowledgments}

The author thanks J.R. Gloudemans as the primary author of Open VSP for producing such a clever and useful tool, Mark Moore for shepherding the development over the years, and Dr. Robert McDonald for his enthusiastic, energetic, and technically excellent support of continued development.

\section{References}

${ }^{1}$ Hahn, A., "Vehicle Sketch Pad: A Parametric Geometry Modeler for Conceptual Aircraft Design", AIAA Paper 2010-657, January 2010.

${ }^{2}$ Box, G.E.P. "Robustness in the Strategy of Scientific Model Building," Robustness in Statistics, edited by R.L. Launer and G.N. Wilkinson, Academic Press, New York, 1979, p. 204.

${ }^{3}$ Courtesy Photograph, "ATT Full Span Model In The 8X6 Foot Wind Tunnel, NASA Identifier: C-1973-2515," http://www.dvidshub.net/image/760029/att-full-span-model-8x6-foot-wind-tunnel\#.UM-YKaWRorn.

${ }^{4}$ Courtesy Photograph, "Pathfinder I With Pressure Wing, W.L. Jones, inspects a transport model between test runs in Langley's National Transonic Facility (NTF)., NASA Identifier: L86-10," http://www.dvidshub.net/image/712066/pathfinderwith-pressure-wing\#.UM-a66WRork.

${ }^{5}$ Courtesy Photograph, "The Double Bubble D8, One of the advanced design concepts, Image credit: NASA/MIT, NASA Identifier: 537761main_d-3-wbwt2," http://www.dvidshub.net/image/724149/double-bubble-d8\#.UM-bsqWRork. 